\title{
Os enunciados da segunda lei da termodinâmica: Uma possível abordagem
}

Statements of the second law of thermodynamics: A possible approach

\author{
R.D.B. Fontana ${ }^{1}$, I.A. dos Santos*2 \\ ${ }^{1}$ Universidade Federal da Fronteira Sul, Campus Chapecó, Chapecó, SC, Brasil \\ ${ }^{2}$ Instituto Federal de Educação, Ciência e Tecnologia da Bahia, Campus Eunápolis, Eunápolis, BA, Brasil
}

Recebido em 27 de agosto de 2015. Aceito em 8 de novembro de 2015

\begin{abstract}
De forma geral os livros texto de física básica abordam a segunda lei da termodinâmica de duas maneiras distintas. Inicialmente, associada à discussão sobre máquinas térmicas, são enunciados os princípios de Clausius e Kelvin; subsequentemente aborda-se a segunda lei introduzindo o conceito de entropia e seu princípio de variaação positiva, ou nula, nas tranformações termodinâmicas. Embora se encontrem demonstrações da equivalência entre os princípios de Kelvin e Clausius, é rara, nos textos básicos de termodinâmica, uma discussão que evidencie a conexão entre os enunciados da segunda lei associados às máquinas térmicas e a abordagem entrópica. Este artigo apresenta uma incursão na segunda lei da termodinâmica com enfoque na equivalência entre seus enunciados, especificamente, entre o princípio da máxima entropia, ou sua variação nunca negativa em qualquer processo termodinâmico, e os princípios associados às máquinas térmicas.
\end{abstract}

Palavras-chave: termodinâmica, segunda lei, equivalência dos enunciados, enunciado entrópico.

The second law of thermodynamics can be found in basic physics textbooks presented in two different ways. Firstly, related to the concept of thermal machines in which the principles of Clausius and Kelvin are postulated. Subsequently, this law is introduced using the concept of entropy and its non negative variation in a thermodynamical transformation (also known as principle of maximum entropy). It is common to find in textbooks the relation between the principles of Clausius and Kelvin of the law, but not so evident the relation between these principles and that one related to variation of the entropy. In this paper we present an incursion into the second law of thermodynamics as seen in these three different ways, specially considering the connection between the second law for entropy variation and as dictated by Clausius and Kelvin.

Keywords: thermodynamics, second law, equivalence between statements, entropy.

\section{Introdução}

O enunciado da segunda lei da termodinâmica é visto em livros didáticos de formas distintas. Em geral é possível encontrar uma formulação atribuída a Kelvin e outra a Clausius, ambas equivalentes por reductio ad absurdum, como discutida, por exemplo, no texto de H.M. Nussenzveig [1]. Esses enunciados se relacionam com as aplicações práticas de motores/refrigeradores do tipo Carnot [2] a partir do qual se estabelece que é impossível para um sistema

*Endereço de correspondência: iasantosif@yahoo.com.br termodinâmico, transformar calor em trabalho integralmente (sem modificar o estado do sistema, pelo menos), ou que é impossível a transferência de calor de uma fonte fria para uma fonte quente sem utilização de trabalho neste processo. Essa visão da segunda lei, como se sabe, acontece no século XIX associada à investigação de máquinas térmicas. Em uma perspectiva mais abrangente do que a dos enunciados que tem relação com máquinas térmicas, podemos encontrar ainda o enunciado relativo à mudança de entropia em um sistema qualquer, considerado também o universo que o rodeia: a entropia 
nunca diminui. Essa segunda abordagem, definida através da mudança na variável de estado, permite estabelecer uma conexão entre a direção preferencial de processos termodinâmicos diversos, tais como a expansão livre de um gás confinado em um recipiente, um processo cuja justificativa seria extremamente complexa se pensada a partir dos enunciados de Clausius ou Kelvin. De fato, a expansão livre de um gás ideal pode ser facilmente demonstrada a respeitar o enunciado da entropia, não tendo relação direta, contudo, com os demais enunciados, pois não é óbvio que um gás ideal nunca passará a ocupar, abruptamente, metade de seu volume, a partir dos enunciados de Kelvin e Clausius.

Na Ref. [2], por exemplo, encontramos primeiramente a segunda lei definida pelo aumento da entropia em processos irreversíveis (e constante em processos cíclicos reversíveis) além de, em sequência, sua definição em termos dos enunciados de Clausius e Kelvin. Há uma conexão entre esses dois últimos enunciados mas não é tão evidente a relação entre o primeiro eunciado e os que o sucedem no texto.

Na Ref. [3], uma reflexão semelhante é feita: as duas maneiras mais comuns de se enunciar a segunda lei da termodinâmica (se com máquinas térmicas, se com entropia) não estão conectadas ao longo do texto, trazendo implicações diferentes e sendo aplicadas a uma vasta gama de problemas de caráter diferentes (o mesmo verifica-se na Ref. [4]).

$\mathrm{Na}$ Ref. [5], a discussão sobre entropia é introduizada a partir da anologia com uma grandeza termodinâmica a qual o leitor já está mais familiarizado, a temperatura. Rememorando que, quando um processo termodinâmico reversível acontece a temperatura constante, o resultado no diagrama pressão versus volume é uma curva denominada isoterma. Assim, a grandeza termodinâmica que permanece constante ao longo de curva isoterma é a temperatura. Argumenta-se que ao se realizar um processo termodinâmico reversível, mantendo-se o sistema isolado termicamente, ou seja, sem que haja troca de calor, o resultado no espaço pressão versus volume será uma curva adiabática e, nesse caso, a grandeza termodinâmica invariante ao longo dessa curva é a entropia. Considerando, portanto, uma isoterma a temperatura $T$, que cruza duas adiabáticas nos pontos $A$ e $B$, a diferença de entropia entre esses pontos será dada pela razão entre o calor trocado e a temperatura da isoterma. A partir deste ponto, por argumentos diferenciais, estabece-se a integral de Clausius. Em termos de demonstração o autor enfatiza a equivalência entre o princípio da máxima entropia e o princípio de mínima energia, além das características dos coeficientes térmicos associados à estabilidade dos sistemas termodinâmicos. Consta ainda ampla discussão dos enunciados de Clausius e Kelvin e suas relações com o princípio de máxima entropia, embora se abstenha de demonstrações de suas equivalências.

De acordo com a Ref. [1], temos as duas formulações equivalentes para a segunda lei, que são aquelas associadas às máquinas térmicas. A conexão entre tais enunciados é relativamente simples e perpassa a noção de que se a negação $A$ implica a negação de $B$ e vice-versa, então necessariamente $A$ implica $B$, sendo essas afirmações a formulação da segunda lei de acordo com Kelvin e Clausius. Há ainda na sequência, por efeito do teorema de Clausius, a introdução da variável de estado entropia, através do que se propõe, por fim, que considerados um sistema termodinâmico qualquer e suas vizinhanças, a soma total da entropia destes deve sempre aumentar. Finalmente encontra-se ainda na referência uma conexão entre o aumento de entropia e o enunciado de Clausius, mas cuja fundamentação não define o que seria um caminho reversível (hipotético) para tal fundamento.

Na Ref. 6] encontramos uma nota motivada por uma lacuna comum nos textos básicos de termodinâmica [1] [, qual seja, a ausência de cálculos generalistas que demonstrem que a variação da entropia é positiva, pelo princípio da máxima entropia, quando dois corpos que possuem temperaturas distintas, inicialmente separados por fronteiras adiabáticas, são colocados em contato até alcançarem o equilíbrio térmico. Em particular, na docência de disciplinas básicas de física, nos parece haver uma falta de conexões mais claras entre os diversos enunciados da segunda lei da termodinâmica, especialmente, desmontrações de equivalência entre os enunciados usalmente associados às máquinas térmicas, Clausius e Kelvin, portanto, e o enunciado entrópico. No presente trabalho propomos uma possibilidade de conexão entre o enunciado de Clausius e o princípio de máxima entropia, a partir do contato térmico entre dois corpos com temperaturas distintas, a fim de se contribuir para ampliar as formas de abordar os enunciados da segunda lei e suas equivalências. 


\section{Segunda lei enunciada}

As formulações dos princípios de Clausius e Kelvin podem ser acessadas em um trabalho do próprio Lord Kelvin, então William Thomson, expressos originalmente da seguinte forma ${ }^{1}[7]$ :

- (C)It is impossible for a self-acting machine, unaided by any external agency, to convert heat from one body to another at a higher temperature.

(É impossível para uma máquina auto-atuant $\AA^{2}$ sem auxílio de um agente externo, transferir calor de um determinado corpo a outro de maior temperatura).

- (K)It is impossible, by means of inanimate material agency, to derive mechanical effect from any portion of matter by cooling it below the temperature of the coldest of the surrounding objects.

(É impossível por meio de um agente inanimado, obter um efeito mecânicd ${ }^{3}$ de qualquer quantidade de matéria através de seu resfriamento abaixo da temperatura do objeto de menor temperatura do entorno).

Na Ref. [8] se vê o princípio de máxima entropia (enunciado de maneira diversa mais adiante em $S$ ) posto em conjunto com a constância da energia do Universo (primeira lei da termodinâmica),

- (S) Die Energie der Welt ist constant. Die Entropie der Welt strebt einem Maximum zu.

(A energia do Universo é constante. A entropia do Universo se direciona a um máximo).

Percebe-se para este enunciadd 4 embora não uma formulação passo a passo, uma argumentação desenvolvida na referência, da qual este emerge como imediata consequência, o que tem o mesmo teor da não negatividade da variação de entropia em um

\footnotetext{
${ }^{1}$ Uma boa versão desses dois princípios em língua portuguesa encontram-se na Ref. [1], a qual será apresentada na sequência. ${ }^{2}$ Cíclica.

${ }^{3} E$. g. realização de trabalho.

${ }^{4}$ (...) dessen vollständige Behandlung hier freilich nicht am Orte sein würde, indem die dazu nötigen Auseinandersetzungen zu umfangreich sein würde. (do qual (tema em questão, o enunciado da entropia) um tratamento completo admite-se não ser encontrado aqui, uma vez que os argumentos necessários seriam demasiado extensos).
}

sistema fechado, o que demonstra o cuidado de Clausius na correlação entre sua primeira versão para a segunda lei e a então emergente da entropia. $\mathrm{Na}$ referência em questão, Clausius perfaz o argumento de se achar um caminho reversível para o cálculo da integral $\int \frac{d Q}{T}=\Delta S$ (a qual é pela primeira vez denominada - variação da - "entropia") ainda que o sistema tenha passado por uma mudança irreversível. Em particular Clausius divide a entropia de um corpo ou sistema em duas partes, uma responsável pela "mudança do conteúdo de calor" e outra relativa a sua "degregação" fazendo alusão à magnitude do grau de separação (desarranjo) das partículas desse corpo.

Para caracterizar a máxima entropia (por conseguinte, sua variação não negativa em sistemas fechados), temos que "Quando, uma transformação em um dado sentido excede, em grandeza, qualquer variação de estadd $7^{7}$ que possa ocorrer no Universo, então o estado do Universo deve se modificar mais do que estas transformações deste primeiro sentido, desta maneira se aproximando, ininterruptamente, de um estado limítrofe $\sqrt{8}$

Na Ref. 9 vemos o mesmo enunciado $\mathbf{C}$ em seu primeiro formato, ainda como consequência das observações de Clausius e do enunciado de Carnot sobre o rendimento de máquinas térmicas.

Na Ref. [10 observa-se também, enunciado por Clausius (na quarta 'coletânea' da Ref. [11]), uma versão equivalente para $C$ : calor nunca pode passar de um corpo mais frio a um mais quente sem que haja outra mudança conectada com este fato, a ocorrer concomitantemente.

Partindo de uma formulação parecida com a Ref. [1] os dois enunciados básicos da lei (de Kelvin $(K)$ e Clausius $(C)$ ) que usaremos são escritos como segue:

- $(K) E$ impossivel realizar um processo cujo único efeito seja a transferência de calor de uma fonte quente para uma fonte fria.

- $(C)$ É impossível realizar um processo cujo único efeito seja remover calor de uma fonte e apro-

\footnotetext{
${ }^{5}$ Disgregation.

${ }^{6}$ Wenn nämlich bei allen im Weltall vorkommenden Zustandsänderungen die Verwandlungen von einem bestimmten Sinne an Grösse übertreffen, so muss der Gesammtzustand des Weltalls sich immer mehr in jenem ersteren Sinne ändern, und das Weltall muss sich somit ohne Unterlass eine Grenzustande nähern.

${ }^{7}$ Espontânea.

${ }^{8}$ de máxima entropia.
} 
veitá-lo integralmente para produção de trabatho.

Como mencionado na introdução, uma equivalência entre essas duas sentenças se vê na Ref. [1] onde o autor demonstra que "não $C$ " implica "não $K$ " assim como "não $K$ " implica "não $C$ ", do que segue imediatamente que $C$ implica $K$ e vice-versa.

Às duas formulações equivalentes acrescenta-se ainda a terceira, em termos das mudanças de entropia em um processo termodinâmico, podendo ser escrita como segue:

- $(S) A$ entropia de um sistema isolado sujeito a um processo termodinâmico sempre cresce (processos irreversíveis) ou permanece constante (processos reversíveis), $\Delta S \geq 0$.

Em [5] vemos, por exemplo, uma relação entre $S$ e $\mathbf{S}$, de acordo com a qual o princípio de variação da entropia nunca a ser negativo em um estado isolado, conduz à máxima entropia quando o equilíbrio é concomitantemente atingido.

Considerando que as demonstrações de equivalência entre $(S)$ e enunciados da segunda lei associados às máquinas términas não ocupam um lugar de destaque nos textos de física básica, neste artigo discutimos uma possibilidade de conexão entre $(S)$ e $(C)$ a partir de um cálculo de troca de calor entre dois corpos a temperaturas distintas.

Tendo em conta apenas a primeira lei da termodinâmica não há o que proíba que, uma vez em contato térmico, o corpo de maior temperatura tenha um incremento nessa e o de menor temperatura um decréscimo na quantidade.

O enunciado $C$ é uma direta negação desse fato, sem contudo estabelecer que, como consequência desse enunciado, existe uma temperatura de equilíbrio tendo o contato térmico se prolongado tempo suficiente. A existência de uma temperatura de equilíbrio parte do enunciado $(S)$, como veremos em sequência, coordenado entre suas duas formas, de máxima entropia e de variação dessa grandeza nunca a ser negativa em um sistema isolado.

\section{Evolução natural de dois corpos em contato térmico: Aumento da entropia}

Partimos de uma situação em que dois corpos de mesma capacidade térmica $\left(C_{1}=C_{2} \equiv C\right)$ e temperaturas distintas $\left(T_{1}<T_{2}\right)$ são colocados em contato térmico, estando isolados do ambiente externo, para o que, a primeira lei da termodinâmica estabelece que $Q_{1}+Q_{2}=0$, o que nos conduz ao resultado, levando em conta que as capacidades térmicas são iguais:

$$
\Delta T_{1}=-\Delta T_{2} .
$$

Perdurando o contato térmico um tempo "pequeno" $\delta t$, as temperaturas evoluem para $T_{a}=T_{1}+\Delta T_{1}$ e $T_{b}=T_{2}+\Delta T_{2}$.

A entropia constitui-se, como introduzida por Clausius, uma função de estado. Portanto, para cada estado de equilíbrio termodinâmico de um sistema há um valor de entropia correspondente. A variação de entropia entre dois estados de equilíbrio só depende dos estados, inicial e final, do sistema e, consequentemente, independe do caminho adotado para o cálculo. Um possível caminho para o cálculo da variação de entropia deste processo pode ser pensado a partir de um processo quasi-estático reversível e isocórico. Para mudar a temperatura de $T_{1}$ até $T_{a}$, o processo reversível corresponde a colocar o corpo em contato térmico com um grande número $N$ de reservatórios térmicos separados cada qual por um $\delta T=\left(T_{a}-T_{1}\right) / N$. Esse processo pode ser invertido colocando o corpo em contato com esses mesmos reservatórios perfazendo um sentido contrário à mudança de $T_{1}$ para $T_{a}$. O mesmo procedimento vale para o segundo corpo que muda sua temperatura de $T_{2}$ para $T_{b}$. Desta forma, podemos calcular a variação da entropia do sistema como:

$$
\begin{aligned}
\Delta S & =\Delta S_{1}+\Delta S_{2}= \\
\int_{1}^{a} \frac{C_{1}}{T} d T & +\int_{2}^{b} \frac{C_{2}}{T} d T=C \ln \left(\frac{T_{a}}{T_{1}} \frac{T_{b}}{T_{2}}\right) .
\end{aligned}
$$

Supondo que $S$ seja válido, temos que

$$
\frac{T_{a} T_{b}}{T_{1} T_{2}} \geq 1
$$

e pela equação da calorimetria (1),

$$
\begin{aligned}
T_{a}\left(T_{2}+T_{1}-T_{a}\right) & \geq T_{1} T_{2}, \\
T_{b}\left(T_{2}+T_{1}-T_{b}\right) & \geq T_{1} T_{2},
\end{aligned}
$$

do que segue

$$
T_{1} \leq T_{a} \leq T_{2}, \quad T_{1} \leq T_{b} \leq T_{2} .
$$

Concluímos, portanto, que após um tempo pequeno $\delta t$, aceitando o enunciado $S$, a temperatura 
do corpo 1 aumentará e do corpo 2 diminuirá. Se esperarmos um tempo suficiente, então, pelo processo descrito acima, eventualmente as temperaturas se encontrarão em um ponto de equilíbrio quando nenhum calor mais é trocado pelos corpos e a entropia é máxima (morte térmica do sistema). Dessa maneira é consequência de $S$ a existência de uma temperatura de equilíbrio e ainda mais, que $T_{1}<T_{e q}<T_{2}$.

Segue imediatamente que se $S$ é falso, $T_{a}<T_{1}$ ou $T_{a}>T_{2}$ após o tempo $\delta t$ e respectivamente $T_{b}>T_{2}$ ou $T_{b}<T_{1}$, o que contradiz $C$ : temos um sistema em que o corpo de maior temperatura teve um incremento desta quando em contato térmico com outro de menor temperatura e esse segundo um decréscimo na grandeza.

Isto nos leva à afirmação de que, de modo análogo ao encontrado na Ref. [1], sendo $C$ falso, $S$ também o será e vice-versa, o que permite associar os dois enunciados: se $S$ é verdadeiro, também o é $C$.

Tal conclusão embora robusta, parte de uma situação em que os corpos tenham capacidade térmicas idênticas. Para uma situação genérica, em que as capacidades térmicas dos corpos são diferentes, a variação de entropia segue uma relação semelhante à Eq. (2), dada por:

$$
\begin{array}{r}
\Delta S=\int_{1}^{a} \frac{C_{1}}{T} d T+\int_{2}^{b} \frac{C_{2}}{T} d T= \\
\ln \left[\left(\frac{T_{a}}{T_{1}}\right)^{C_{1}}\left(\frac{T_{b}}{T_{2}}\right)^{C_{2}}\right],
\end{array}
$$

e de acordo ainda com a primeira lei, pelo estudo da calorimetria temos:

$$
C_{1} \Delta T_{1}=-C_{2} \Delta T_{2}
$$

Supondo que o enunciado $S$ esteja correto, devemos assumir que:

$$
\left(\frac{T_{a}}{T_{1}}\right)^{C_{1}}\left(\frac{T_{b}}{T_{2}}\right)^{C_{2}} \geq 1 .
$$

Provaremos a seguir que $C$ é válido como decorrência da validade dessa inequação e como consequência que quando $S$ não é válido também $C$ não o é, tornando sem efeito o enunciado $K$, igualmente. Para tal prova, supomos inicialmente as seguintes relações:

$$
\delta \equiv \frac{C_{1}}{C_{2}} ; \quad \alpha \equiv \frac{T_{a}}{T_{1}} ; \quad \beta \equiv \frac{T_{b}}{T_{2}} ; \quad \gamma \equiv \frac{T_{1}}{T_{2}} .
$$

Nesse caso é sempre válido que $\delta, \alpha, \beta, \gamma>0$ e em particular para nosso caso que $\gamma<1$. Da Eq. (7) segue imediatamente com os coeficientes definidos na Eq. $(9)$ que $\delta=\frac{1}{\gamma}\left(\frac{1-\beta}{\alpha-1}\right)$, o que, levado em consideração conjuntamente com a desigualdade $\delta>0$ resulta em

$$
\frac{1-\beta}{\alpha-1}>0
$$

A relação (10) nos conduz à importante suposição de duas condições mutuamente excludentes $(C E)$, a saber:

(i) $\alpha<1$, resulta necessariamente em $\beta>1$, ou;

(ii) $\alpha>1$ leva à $\beta<1$.

Isso significa que, ainda que não consideremos a segunda lei da termodinâmica, a única evolução para o sistema, ao colocarmos dois corpos com temperaturas diferentes em contato térmico (de acordo com a primeira lei da termodinâmica) é aquela em que a temperatura de um dos corpos se eleva e de outro diminui (estando ambos impedidos de elevar - ou abaixar - concomitantemente suas temperaturas).

Resta, portanto, provar que com a introdução da segunda lei (lida através do enunciado $S$ ), na situação dada pela inequação (8) e as condições anteriores, necessariamente $\alpha>1$ e $\beta<1$, ou seja, uma vez em contato térmico, o aumento da entropia dos corpos estabelece a aproximação de suas temperaturas até que o equilíbrio seja estabelecido (se o contato térmico durou tempo suficiente).

Tomando a inequação (8), elevada à potência $1 / C_{2}$ obtemos:

$$
\beta \alpha^{\frac{1}{\gamma}\left(\frac{1-\beta}{\alpha-1}\right)} \geq 1,
$$

e para $\beta>0$,

$$
\alpha^{\frac{1}{\gamma}\left(\frac{1-\beta}{\alpha-1}\right)} \geq \frac{1}{\beta} .
$$

Para a inequação acima deve valer uma das duas condições $(C E)$. De fato ao supormos $(i)$ como verdadeira, conseguimos, considerando o logaritmo na base $\alpha$,

$$
\frac{1}{\gamma}\left(\frac{1-\beta}{\alpha-1}\right) \leq \log _{\alpha} \frac{1}{\beta}=-\frac{\ln \beta}{\ln \alpha}
$$

sendo o lado direito da inequação positivo (devemos observar que a relação da desigualdade se inverte visto que $\alpha<1$, ou seja, $\log \alpha<0$ ). Podemos multiplicar esta inequação por $-\gamma \frac{\ln \alpha}{\ln \beta}$, já que $\gamma>0$ 
obtendo:

$$
\begin{gathered}
\frac{\ln \alpha}{\ln \beta}\left(\frac{\beta-1}{\alpha-1}\right) \leq \gamma<1 \rightarrow \\
\left(\frac{\ln \alpha}{\alpha-1}\right)\left(\frac{\beta-1}{\ln \beta}\right)<1 .
\end{gathered}
$$

Dessa maneira para que as temperaturas evoluam em sentido contrário ao esperado e a entropia diminua (invalidando os enunciados $C$ e $S$ diretamente), a inequação (14) deve ser válida (além da condição $(i))$. Para sua análise, supomos as seguintes funções e domínios

$$
\begin{aligned}
& F(\alpha)=\frac{\ln \alpha}{\alpha-1} \rightarrow 0<\alpha<1 \\
& G(\beta)=\frac{\beta-1}{\ln \beta} \rightarrow \beta>1 .
\end{aligned}
$$

Demonstraremos a seguir que tanto $F$ quanto $G$ são maiores do que 1 no domínio especificado acima.

Para $F$ temos

$$
\begin{aligned}
\lim _{\alpha \rightarrow 1} F(\alpha) & =\lim _{\alpha \rightarrow 1} \frac{\partial_{\alpha}(\ln \alpha)}{\partial_{\alpha}(\alpha-1)}= \\
\lim _{\alpha \rightarrow 1} \frac{1 / \alpha}{1} & =1 \\
\frac{\partial F}{\partial \alpha} & =\frac{\alpha(1-\ln \alpha)-1}{\alpha(\alpha-1)^{2}} \equiv \frac{P(\alpha)}{Q(\alpha)} \\
\lim _{\alpha \rightarrow 1} \frac{\partial F}{\partial \alpha} & =\lim _{\alpha \rightarrow 1} \frac{-\ln \alpha}{(\alpha-1)(3 \alpha-1)}= \\
\lim _{\beta \rightarrow 1} \frac{-1 / \alpha}{6 \alpha-4} & =-\frac{1}{2} \\
\frac{\partial P}{\partial \alpha} & =-\ln \alpha .
\end{aligned}
$$

Pela (17), vemos que $F$ tem valor unitário no limite de seu domínio, $\alpha \rightarrow 1$. Vemos ainda que neste ponto limítrofe, a derivada assume um valor negativo 20. Também a função $Q=\alpha(\alpha-1)^{2}$ é sempre positiva nesse domínio. Por sua vez a função $P=\alpha(1-\ln \alpha)-1$ tem um limite dado por $P(1)=0$, sendo sempre crescente até esse limite, pois sua derivada expressa em (20) é sempre positiva. Disso segue que $P<0$ no intervalo $0<\alpha<1$ e $Q>0$. Finalmente, levando em consideração a (18), vemos que a derivada de $F$ é sempre negativa no intervalo $0<\alpha<1$ (pelos sinais de $Q$ e $P$ serem opostos). Sendo sua derivada negativa em todo domínio, percebemos que $F$ é decrescente até seu valor limítrofe $F(\alpha \rightarrow 1)=1$, ou seja, $F>1$ em todo seu domínio.

A situação para a função $G$ é análoga à apresentada acima e pode ser extraída das relações:

$$
\begin{aligned}
& \lim _{\beta \rightarrow 1} G(\beta)=\lim _{\beta \rightarrow 1} \frac{\partial_{\beta}(\beta-1)}{\partial_{\beta}(\ln \beta)}= \\
& \lim _{\beta \rightarrow 1} \frac{1}{1 / \beta}=1, \\
& \frac{\partial G}{\partial \beta}=\frac{\beta(\ln \beta-1)+1}{\beta \ln ^{2} \beta} \equiv \frac{A(\beta)}{B(\beta)}, \\
& \lim _{\beta \rightarrow 1} \frac{\partial G}{\partial \beta}= \\
& \lim _{\beta \rightarrow 1} \frac{(\ln \beta-1)+\beta(1 / \beta)}{\ln ^{2} \beta+2 \beta(\ln \beta) / \beta}=\frac{1}{2}, \\
& \frac{\partial A}{\partial \beta}=\ln \beta .
\end{aligned}
$$

Pela Eq. (21), $G$ tem limite unitário no ponto $\beta=1$. De fato $G$ é sempre crescente uma vez que sua derivada é sempre positiva no domínio $\beta>1$. Isso pode ser visto a partir da Eq. (22) com as relações para $A=\beta(\ln \beta-1)+1$ e $B=\beta \ln ^{2} \beta$ : $B>0$ sempre que $\beta>1$. Quanto a $A$, pela Eq. (24), percebemos que sua derivada é sempre positiva para $\beta>1$, ou seja, a partir de $A(\beta=1)=0$, essa função é positiva. Isso significa que a partir do ponto $\left.\frac{\partial G}{\partial \beta}\right|_{\beta \rightarrow 1}=\frac{1}{2}>0$, a derivada de $G$ é sempre positiva, do que se segue que $G$ é crescente em todo domínio. Assim concluímos que $G>1$ neste domínio, com limite dado pela Eq. 21).

Um gráfico das funções $G$ e $F$ segue na Fig. 1.

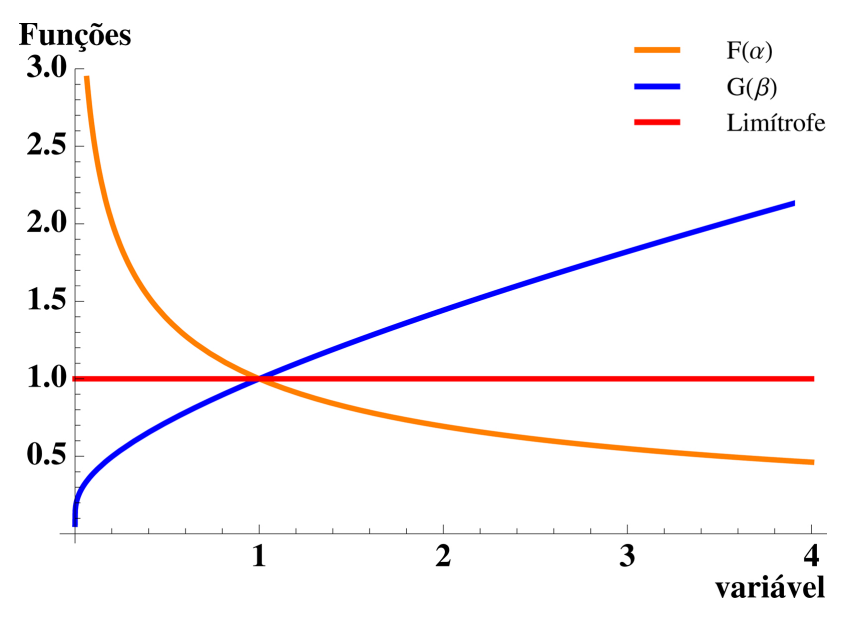

Figura 1: Funções $G(\beta)$ e $F(\alpha)$ maiores do que 1 no domínio especificado pela condição $(i)$. 
Com as relações para $G$ e $F$, mostramos que:

$$
F(\alpha) G(\beta)=\left(\frac{\ln \alpha}{\alpha-1}\right)\left(\frac{\beta-1}{\ln \beta}\right)>1 .
$$

Disso segue imediatamente que a condição $(i)$ invalida a inequação (11), ou seja, o enunciado do aumento da entropia só pode valer se a condição (ii) for válida: as temperaturas devem se aproximar independentemente do valor das capacidades específicas dos corpos (como se observa). De fato, a condição $(i)$ adquire sentido apenas quando $\left(\frac{\ln \alpha}{\alpha-1}\right)\left(\frac{\beta-1}{\ln \beta}\right)>1$, ou seja, quando $T_{1}>T_{2}$. Isto significaria, neste caso, uma aproximação das temperaturas a partir do contato térmico. Então o aumento da entropia do sistema indica necessariamente uma aproximação das temperaturas dos corpos colocados em contato térmico.

Fica evidente, portanto, que o aumento da separação entre as temperaturas dos corpos (negação de $C$ ) diminui a entropia (negação de $S$ ): a desordem é máxima quando o equilíbrio é atingido. Equivalentemente, se as temperaturas se aproximam, a entropia aumenta.

Disso segue imediatamente a emergência de uma temperatura de equilíbrio: se a entropia varia sempre em direção a um máximo, esse máximo será representado necessariamente pelo estado de equilíbrio quando nenhum calor pode ser trocado pelos corpos. Essa conclusão pode ser obtida de maneira relativamente simples com a variação de entropia do sistema dada na Eq. (6):

$$
S=S_{0}+C_{2} \ln \left[\left(\frac{T_{a}}{T_{1}}\right)^{C_{1} / C_{2}}\left(\frac{T_{b}}{T_{2}}\right)\right] .
$$

Levando em conta a primeira lei dada na Eq. (7), temos:

$$
\frac{S}{C_{2}}=\frac{S_{0}}{C_{2}}+\left(\frac{T_{2}-T_{b}}{T_{a}-T_{1}}\right) \ln \left(\frac{T_{a}}{T_{1}}\right)+\ln \left(\frac{T_{b}}{T_{2}}\right),
$$

e ainda,

$$
\begin{array}{r}
\frac{\partial S}{\partial T_{a}}=C_{2}\left[\frac{T_{2}-T_{b}}{T_{a}\left(T_{a}-T_{1}\right)^{2}}\right] \times \\
{\left[T_{a}-T_{1}-T_{a} \ln \left(\frac{T_{a}}{T_{1}}\right)\right],} \\
\frac{\partial S}{\partial T_{b}}=C_{2}\left[\frac{1}{T_{b}\left(T_{a}-T_{1}\right)}\right] \times \\
{\left[T_{b}-T_{1}-T_{b} \ln \left(\frac{T_{a}}{T_{1}}\right)\right] .}
\end{array}
$$

A máxima entropia é atingida quando a variação dessa função é nula, ou seja, pelas equações acima, quando:

$$
\begin{array}{r}
T_{a}-T_{1}-T_{a} \ln \left(\frac{T_{a}}{T_{1}}\right)= \\
0=T_{b}-T_{1}-T_{a} \ln \left(\frac{T_{a}}{T_{1}}\right),
\end{array}
$$

ou seja, quando $T_{a}=T_{b}$.

\section{Conclusões}

A partir do contato térmico entre dois corpos, isolados do meio externo, de temperaturas incialmente diferentes e capacidades térmicas iguais, demonstrouse que o enunciado entrópico da segunda lei da termodinâmica implica que a evolução espontânea do sistema resultará em uma temperatura de equilíbrio $\left(T_{1}<T_{e q}<T_{2}\right)$ entre os corpos concomitantemente a uma máxima entropia do sistema. A generalização dessa prova para corpos de capacidades térmicas distintas mostrou que embora o princípio de Joule (primeira lei da termodinadâmica) garanta que a evolução do sistema é tal que um dos corpos tem sua temperatura elevada enquanto para o outro corpo haverá diminuição, apenas a segunda lei assegura que essa evolução será no sentido aproximação das temperaturas, resultando em uma temperatura de equilíbrio para o sistema, após transcorrido tempo suficiente. A demonstração de equivalência entre os enunciados de Clausius e o princípio de máxima entropia oferece uma alternativa de abordagem para a discussão das formulações da segunda lei da termodinâmica e suas implicações.

\section{Agradecimentos}

Os autores agradecem ao CNPq por suporte financeiro. I.A. dos Santos agradece à FAPESB pelo apoio financeiro.

\section{Referências}

[1] H. Moysés Nussenzveig, Curso de Física Básica Vol. 2, (Editora Blucher, 2002), $4^{\mathrm{a}}$ ed.

[2] D. Halliday, R. Resnick, J. Walker, Fundamentos de Física Vol. 2 (LTC - Livros Técnicos e Científicos Editora S.A., Rio de Janeiro, 2009), 8 a ed.

[3] R. A. Serway, J. W. Jewett, Princípios de Física Vol. 2, (Cengage Learning, 2004), $3^{\mathrm{a}}$ ed. 
[4] P. A. Tipler, Física para engenheiros e cientistas Vol. 1, (LTC - Livros Técnicos e Científicos Editora S.A., Rio de Janeiro, 2009), $6^{\mathrm{a}}$ ed.

[5] M. J. de Oliveira, Termodinâmica, (Editora Livraria da Física, 2005).

[6] F. M. S. Lima, Change of entropy in the spontaneous thermalization of two distinct blocks, Revista Brasileira do Ensino de Física 37, n 1, 1701 (2015).

[7] W. Thomson, On the dynamical theory of heat, with numerical results deduced from Mr. Joule's equivalent of a thermal unit, and M. Regnault's observations on steam, Philosophical Magazine 4, 174 (1852).

[8] R. Clausius, Über verschiedene für die Anwendug bequeme Formen der Hauptgleichungeng der mechanischen Wärmetheorie, Annalen der Physik und Chemie 125, 353-400 (1865).

[9] R. Clausius, Über die bewegende Kraft der Wärme und die Gesetze, welche sich daraus für die Wärmelehre selbst ableiten lassen, Annalen der Physik und Chemie 155, 368-397 (1850).

[10] R. Clausius, Über eine veränderte Form des zweitens Hauptsatzes der mechanischen Wärmetheorie, Annalen der Physik, (1854).

[11] R. Clausius, The mechanical theory of heat, with its application to the steam-engine and to the physical properties of the bodies, (Ed. T. Archer Hirst, London, 1867). 